\title{
BMJ Open Chest pain in general practice: a systematic review of prediction rules
}

\author{
Ralf E Harskamp, ${ }^{\oplus 1,2}$ Simone C Laeven, ${ }^{1}$ Jelle CL Himmelreich, \\ Wim A M Lucassen, ${ }^{1}$ Henk C P M van Weert ${ }^{1}$
}

To cite: Harskamp RE, Laeven SC, Himmelreich JCL, et al. Chest pain in general practice: a systematic review of prediction rules. BMJ Open 2019;9:e027081. doi:10.1136/ bmjopen-2018-027081

- Prepublication history and additional material for this paper are available online. To view these files, please visit the journal online (http://dx.doi. org/10.1136/bmjopen-2018027081).

Received 4 October 2018 Revised 27 November 2018 Accepted 18 December 2018

Check for updates

(c) Author(s) (or their employer(s)) 2019. Re-use permitted under CC BY-NC. No commercial re-use. See rights and permissions. Published by BMJ.

1Department of General Practice, Amsterdam UMC, University of Amsterdam, Amsterdam Public Health, Academic Medical Center, Amsterdam, The Netherlands

${ }^{2}$ Duke Clinical Research Institute, Durham, North Carolina, USA

Correspondence to

Dr. Ralf E Harskamp; r.e.harskamp@gmail.com

\section{ABSTRACT \\ Objective To identify and assess the performance of clinical decision rules (CDR) for chest pain in general practice.}

Design Systematic review of diagnostic studies. Data sources Medline/Pubmed, Embase/Ovid, CINAHL/ EBSCO and Google Scholar up to October 2018.

Study selection Studies that assessed CDRs for intermittent-type chest pain and for rule out of acute coronary syndrome (ACS) applicable in general practice, thus not relying on advanced laboratory, computer or diagnostic testing.

Review methods Reviewers identified studies, extracted data and assessed the quality of the evidence (using Quality Assessment of Diagnostic Accuracy Studies (QUADAS-2)), independently and in duplicate.

Results Eight studies comprising five CDRs met the inclusion criteria. Three CDRs are designed for rule out of coronary disease in intermittent-type chest pain (Gencer rule, Marburg Heart Score, INTERCHEST), and two for rule out of ACS (Grijseels rule, Bruins Slot rule). Studies that examined the Marburg Heart Score had the highest methodological quality with consistent sensitivity $(86 \%-$ $91 \%)$, specificity $(61 \%-81 \%)$ and positive $(23 \%-35 \%)$ and negative (97\%-98\%) predictive values (PPV and NPV). The diagnostic performance of Gencer (PPV: $20 \%-34 \%$, NPV: 95\%-99\%) and INTERCHEST (PPV: 35\%-43\%, NPV: 96\%-98\%) appear comparable, but requires further validation. The Marburg Heart Score was more sensitive in detecting coronary disease than the clinical judgement of the general practitioner. The performance of CDRs that focused on rule out of ACS were: Grijseels rule (sensitivity: 91\%, specificity: 37\%, PPV: 57\%, NPV: $82 \%$ ) and Bruins Slot (sensitivity: 97\%, specificity: 10\%, PPV: 23\%, NPV: $92 \%)$. Compared with clinical judgement, the Bruins Slot rule appeared to be safer than clinical judgement alone, but the study was limited in sample size.

Conclusions In general practice, there is currently no clinical decision aid that can safely rule out ACS. For intermittent chest pain, several rules exist, of which the Marburg Heart Score has been most extensively tested and appears to outperform clinical judgement alone.

\section{INTRODUCTION}

Chest pain is a common symptom for contacting the general practitioner (GP). During office hours, $1.5 \%$ of all consultations and $4 \%$ of all new episodes are related to chest pain. ${ }^{1-5}$ The highest frequency of chest
Strengths and limitations of this study

The study provides an up-to-date overview on chest pain rules applicable in general practice.

- We applied stringent inclusion criteria and standardised quality assessment tools.

- Various diagnostic study designs were included (ie, derivation, validation)

- Chest pain rules that relied on advanced diagnostic testing (ie, HEART, TIMI or GRACE) were not included.

- Decision rules based on exclusively non-chest pain symptoms (ie, dyspnoea) were not part of the literature search.

pain consultations is in the age category 45 to 64 years, with notable differences between men and women in its presentation. ${ }^{13} 46$ The initial task for GPs is differentiating less frequent but urgent diagnoses of chest pain, such as acute coronary syndrome, or pulmonary embolism, from more common but less urgent diagnoses (such as gastro-oesophageal reflux, musculoskeletal pain or anxiety). ${ }^{1-5}$ To make this important differentiation, GPs mainly depend on history taking, medical history, physical examination and past experience to establish a working hypothesis/diagnosis. The most prevalent reason for referral is rule out of acute coronary syndrome (ACS) in patients with acute-onset chest pain as well as rule out of coronary artery disease (CAD) in patients who present with intermittent-type chest pain.

The GPs' evaluation of patients with chest pain, based on symptoms and signs alone ('clinical gestalt'), is unfortunately insufficient for diagnosing or excluding stable angina and particularly ACS reliably (sensitivity of $69 \%$ and specificity of $89 \%) .{ }^{7}$ GPs are very well aware of their own limitations and therefore apply a low referral threshold. A validated clinical risk score could aid GPs in decision-making by calculating the risk of an unfavourable diagnosis based on patient characteristics, symptoms and other readily available information. In this systematic review, 
we aim to identify and assess the performance of existing clinical decision aids/rules for stable angina and/or acute coronary syndrome in patients with chest pain that are applicable and have been validated in low-resource general practice or equivalent settings.

\section{METHODS}

The Preferred Reporting Items for Systematic Reviews and Meta-Analyses (PRISMA) guidelines were used to undertake this review. ${ }^{8}$

\section{Data sources and searches}

We searched PubMed, Embase, CINAHL and Google Scholar from database inception through to the search date 17 October 2018. We searched for studies written in English, Dutch or German. We used keywords: chest pain, coronary artery disease, acute coronary syndrome, general practice, primary care practice, prediction rule, decision model or decision aid. Online supplement A of the supplemental data document displays the full search strategy.

\section{Study selection}

Two investigators (REH, SCL) identified potentially eligible studies, with a third (WAML) to resolve any disagreements. We used an online systematic review platform (Covidence, Veritas Health Innovation, Melbourne, Australia) for this purpose. In addition to the language (English, Dutch, German) and human research restrictions, the following inclusion criteria for eligible studies were applied: (1) original studies in adults ( $\geq 18$ years of age) with enrolment in a primary care setting; (2) chest pain either acute or intermittent-type; (3) ascertainment of the diagnosis of coronary artery disease or acute coronary syndrome at follow-up; (4) predictive tool based on multivariable analysis; (5) predictive tool derived from findings that are applicable in primary care setting. These findings may include: medical history, physical examination, ECG or previously documented laboratory findings (such as lipid levels). We excluded studies with a retrospective study design and studies that used a prediction rule that was based on serial biomarker testing (ie, sequential troponin testing at 2-3-hour time interval), required advanced computer algorithms or advanced diagnostic testing (cardiac imaging, coronary angiography).

\section{Clinical decision rules and outcomes of interest}

The clinical decision aids may include items from history taking, physical examination, laboratory and electrocardiographic data. The outcomes of interest are diagnostic test characteristics of included rules, including: sensitivity, specificity, negative and positive prediction values.

\section{Reference diagnosis}

The clinical outcomes that we used as reference diagnosis were (1) any form of coronary artery disease (CAD) or coronary heart disease; or (2) a more restricted form including unstable angina or myocardial infarction (referred to as acute coronary syndrome) in patients with acute chest pain. We applied no restrictions on minimum or maximum time of follow-up. The assessment of applicability of the reference standard for each study is assessed by the QUADAS-2 tool, which can be found as supplemental data in online supplement $B$.

\section{Study population}

We included studies with adult populations that present at a GP office or out-of-office setting (ie, patient visits when making house calls). In-hospital, emergency department (ED) and/or preselected outpatient populations were not eligible.

\section{Data extraction and quality assessment}

Two investigators (REH, SCL) extracted data elements from each study, with a third investigator (WAML) independently reviewing these data for accuracy. The quality of the studies was assessed by three investigators (REH, SCL, WAML) using the Quality Assessment of Diagnostic Accuracy Studies (QUADAS-2) tool for assessing risk of bias in diagnostic accuracy studies. This tool comprises four key domains, namely: patient selection, index test, reference standard and flow and timing. ${ }^{9}$ We assessed whether a clinical decision rule (CDR) was ready for application in clinical practice based on the level of evidence for each rule using the definitions of the Mount Sinai Evidence-Based Medicine Working group. ${ }^{10}$

\section{Data synthesis and analysis}

The extracted data on study and patient characteristics, outcome measures and follow-up information of the included studies will be displayed in tables. Subsequently, we extracted data on the discriminatory properties (C-statistic) of the decision rule from each studies, as well as data on sensitivity, specificity, positive (PPV) and negative predictive values (NPV) and true and false positives and negatives. We constructed a summary receiver operating characteristic curve based on $2 \times 2$ tables from the individual study data using Review Manager (RevMan V.5.3. The Cochrane Collaboration, Copenhagen, Denmark).

\section{Patient and public involvement}

This study did not involve direct patient involvement. For the current analysis, we did not a priori consult with representatives of patient organisations. After peer review and acceptance of publication, we will share the findings of our research with the Dutch Heart Foundation, relevant patient organisations, as well as general practitioners within our academic network.

\section{RESULTS}

\section{Search results}

Our search resulted in 3105 unique studies of which we assessed 94 in full text. Of those, eight studies met the inclusion criteria of our study, in which five different CDRs were evaluated. All studies were written in English. 
3,105 studies screened

3,011 studies irrelevant

94 full-text studies assessed for eligibility

86 studies excluded
43 wrong population (ED, hospital)
15 wrong study design (case report, review, etc)
14 no clinical decision rule
7 rule requires use of computer (algorithm)
3 rule not derived from multivariable analysis
3 wrong outcome parameter (no involving CAD/ACS)
1 No clinical parameters included in rule

\section{8 studies included}

Figure 1 Flow chart of systematic search of the literature. ACS, acute coronary syndrome; CAD, coronary artery disease; ED, emergency department.

The flowchart of our search strategy and reasons for exclusions can be found in figure 1 .

\section{Quality assessment}

The overall quality of the studies was moderate as graphically displayed in figure 2. In six out of eight studies, we found a high risk of bias in the reference standard, as the assessors who determined the final diagnosis (delayed type) were not blinded to the index test results. In three studies, we found a high risk of bias in patient selection as a significant proportion of patients were excluded prior to enrolment. ${ }^{11-13}$ Also, in four studies, a high risk of bias was found in flow and timing, due to relatively high dropout rates of patients. ${ }^{72-14}$ In one study, $>15 \%$ of participating GPs stopped recruiting prematurely. ${ }^{7}$ Quality concerns of the pooled individual data study (INTERCHEST) included possible bias due to missing data in $>20 \%$ of the study population and unverifiable risks of bias regarding patient selection. ${ }^{14}$ Details of the quality assessment can be found as online supplement B in the supplemental data file.

Risk of bias

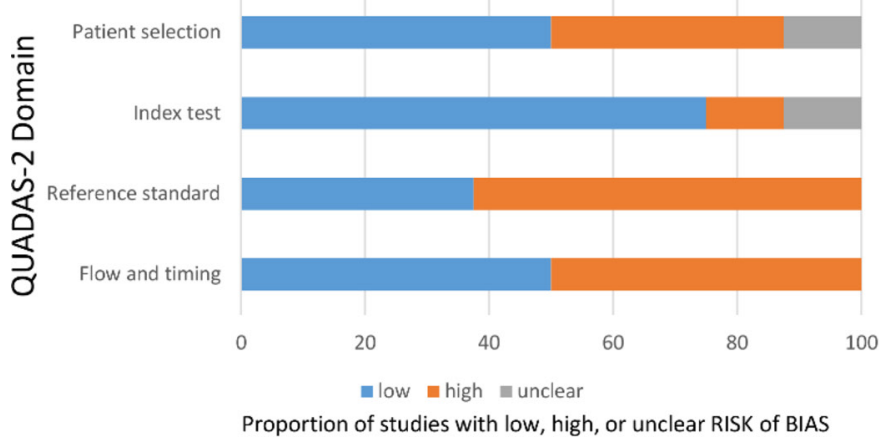

Figure 2 Quality assessment by QUADAS-2.

\section{Study and patient characteristics}

As shown in table 1, a total of seven single cohort studies were included involving 6959 patients and one pooled individual data study from five cohorts (INTERCHEST) involving 3099 patients. The sample size of the individual cohort studies ranged from 289 to 1249 patients. Studies were conducted in Europe and the USA and were published 1995 and 2017. All studies were conducted in general practice, with two studies mandating immediate work-up of all patients at the ED. ${ }^{12} 13$ The prevalence of CAD, with a variable diagnostic follow-up period of up to 1 year, ranged from $8.0 \%$ to $15.0 \%$. In three studies concentrating on acuteonset chest pain, the prevalence of ACS ranged from $22.0 \%$ to $47.8 \% .^{11-13}$ The reported mean age of patients ranged from 41 to 67 years, with women comprising $44 \%$ to $58 \%$ of the population. In studies that reported the prevalence of comorbidities, hypertension (45\%$50 \%)$ and dyslipidaemia $(31 \%-41 \%)$ were common, and diabetes was present in approximately $13 \%$. The inclusion and exclusion criteria as well as the definitions that were used for the reference diagnoses for each of the studies can be found as online supplement $\mathrm{C}$ and D in the supplemental file.

\section{Clinical decision rules}

We identified a total of five CDRs, namely the Gencer rule $^{7}$ the Marburg Heart Score, ${ }^{15-17}$ INTERCHEST, $^{14}$ Grijseels rule ${ }^{12}{ }^{13}$ and Bruins Slot rule. ${ }^{11}$ As shown in table 2 , the CDRs have been developed based on readily available clinical information, such as patient characteristics, medical history and physical examination. The Grijseels rule also requires an ECG. The former three scores (Gencer, Marburg Heart Score and INTERCHEST) were developed for rule out of CAD, whereas the Grijseels and Bruins Slot rules were constructed for rule out of ACS.

\section{Decision rules for stable coronary artery disease in patients with intermittent chest pain}

As shown in table 3, the decision aid that was most extensively tested is the Marburg Heart Score. This

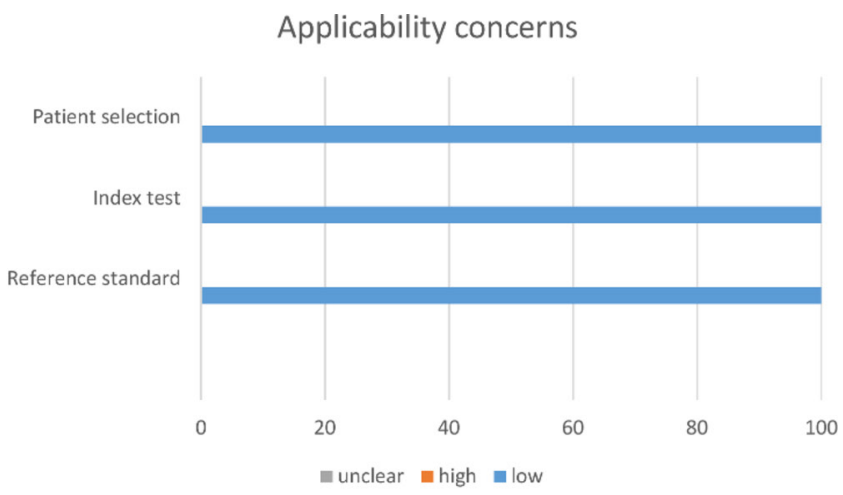

Proportion of studies with low, high, or unclear CONCERNS regarding APPLICABILITY 
Table 1 Characteristics of the study design and study population

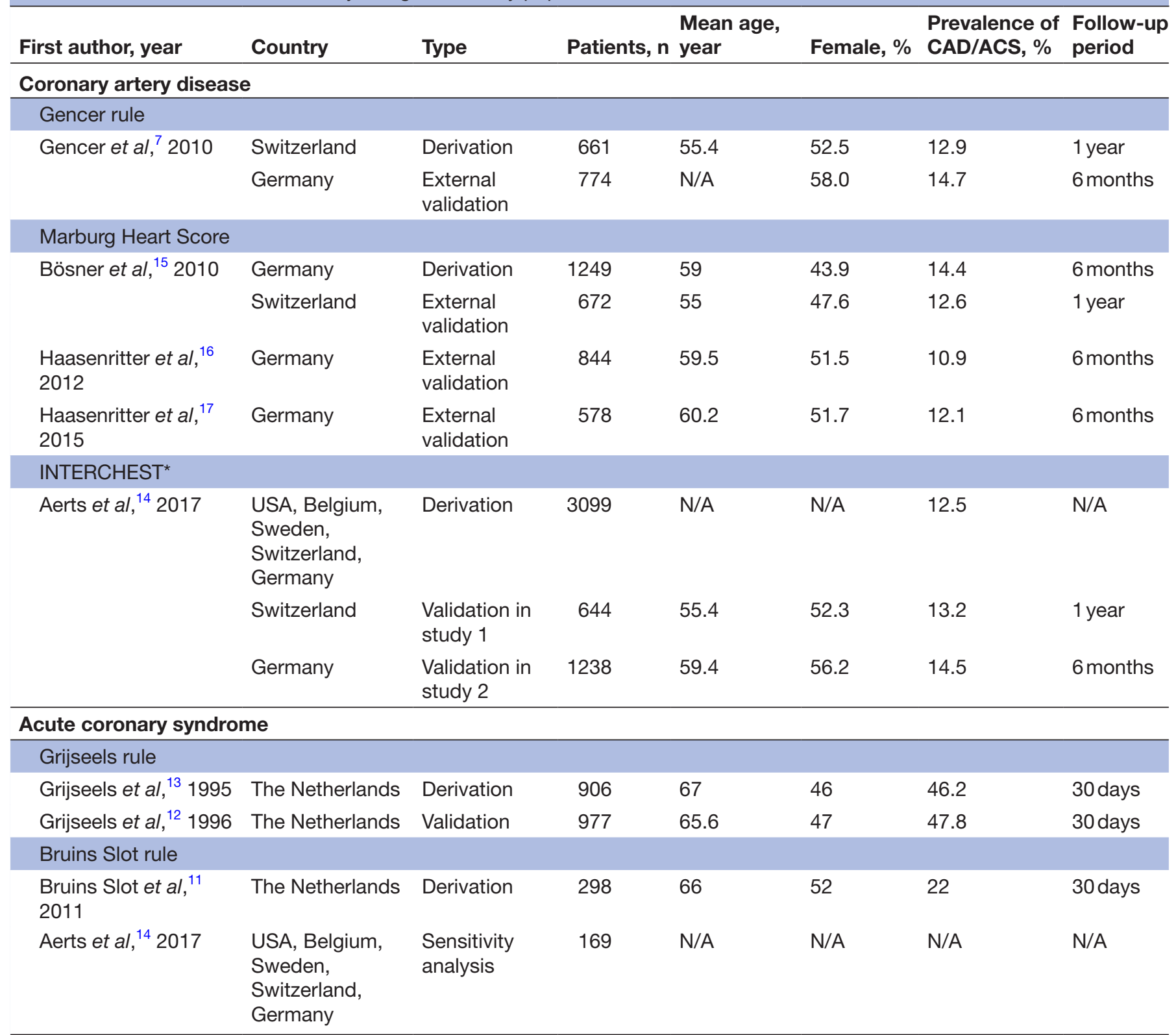

${ }^{*}$ Derivation used pooled individual patient data from five studies. The INTERCHEST was applied to two of these five studies to measure its diagnostic performance. We referred to this as 'validation in study 1 and 2'.

ACS, acute coronary syndrome; CAD, coronary artery disease; N/A, not applicable.

study has good overall discrimination (C-statistic of $0.84-0.90)$, with a sensitivity of $86 \%-89 \%$, specificity of $64 \%-81 \%$, with a PPV of $23 \%-40 \%$ and a NPV of $97 \%-98 \%$. The diagnostic properties of the Marburg Heart Score are visualised in figure 3, illustrating its consistent diagnostic performance in terms of sensitivity and specificity. As shown in table 4, The Marburg Heart Score was found to outperform unaided clinical judgement. When used as a decision aid, both the sensitivity $(+8.0 \%)$ and specificity were higher $(+5.8 \%)$. Moreover, when the Marburg Heart Score was used for an initial triage tool, it led to higher specificity $(+11.6 \%)$ with similar sensitivity $(-1.5 \%)$ compared with unaided clinical judgement. Based on the combined body of evidence, the level of evidence is 2 for the Marburg Heart Score, which implicates that this rule can be used in a general practice setting of low-risk patients with intermittent chest pain with confidence in its accuracy.

The other two CDRs for rule out of stable CAD were the INTERCHEST rule and the Gencer rule. The INTERCHEST rule which was derived from a pooled data analysis also shows promise (C-statistic of 0.84 , sensitivity $82 \%-88 \%$, specificity $74 \%-82 \%$, PPV of $35 \%-43 \%$ and NPV of $96 \%-98 \%$ ), but has a number of quality concerns, and has not been compared with unaided clinical judgement. As such, the INTERCHEST rule should not be considered ready for clinical 
Table 2 Components of the clinical decision rules

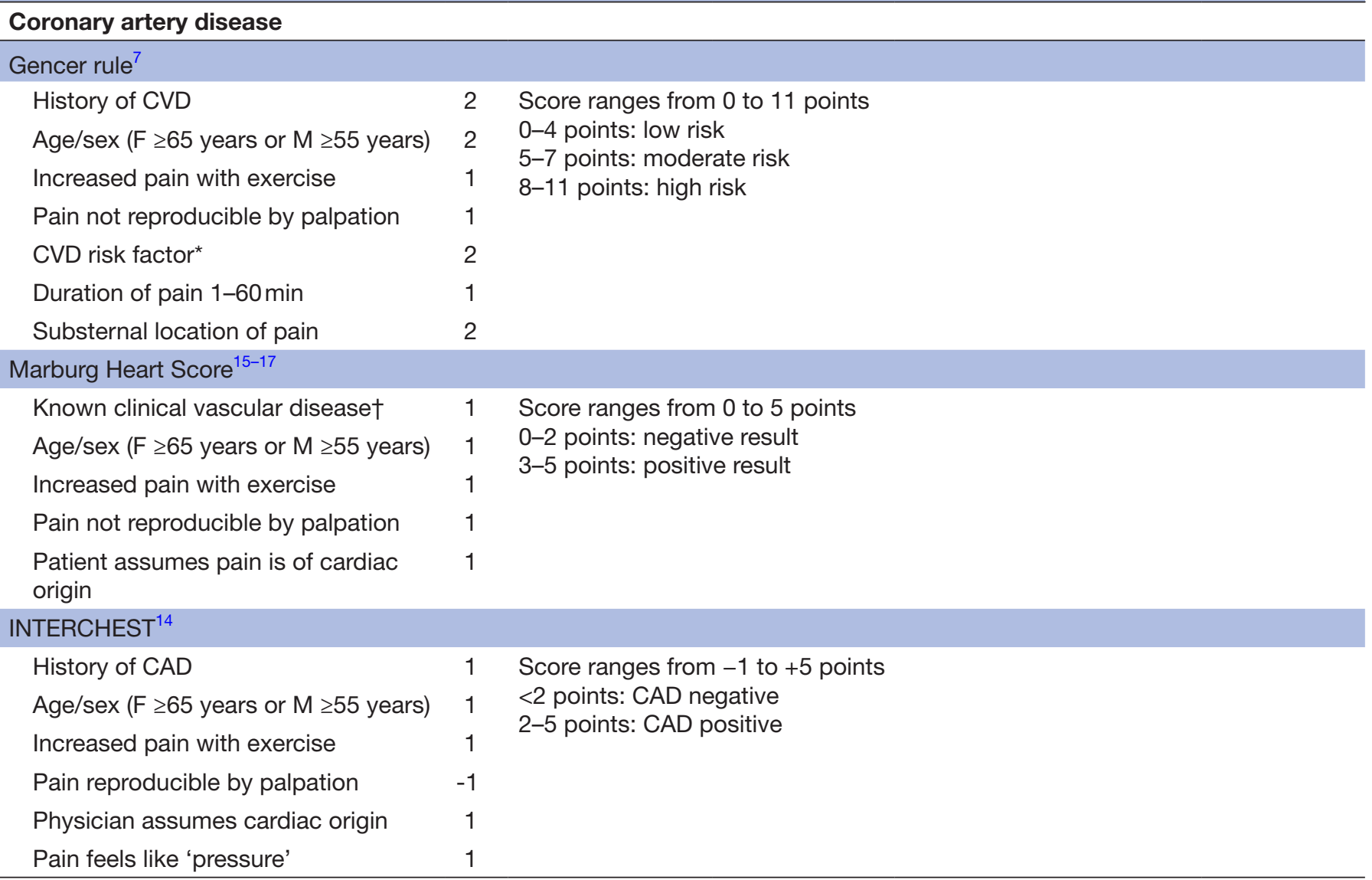

\section{Acute coronary syndrome}

\begin{tabular}{|c|c|c|c|}
\hline Grijseels rule r $^{1213}$ & & & \\
\hline History of CAD & Variables present Normal ECG & Possible/minor MI on ECG & $\begin{array}{l}\text { Major MI on } \\
\text { ECG }\end{array}$ \\
\hline
\end{tabular}

$\begin{array}{llll}\text { Male sex } & 0 & \text { Home } & \text { Possible referral Always }\end{array}$ referral and start treating as ACS

$\begin{array}{llll}\text { Presence of radiation of pain } & 1 & \text { Home } & \text { Referral } \\ \text { Presence of nausea/sweating } & 2 & \text { Possible referral Referral } \\ \text { Abnormal ECG } & >=3 & \text { Referral } & \text { Referral }\end{array}$

\begin{tabular}{lll} 
Bruins Slot rule $^{11}$ & & \\
History of CAD & 2 & Score ranges from 0 to 20 points \\
Male sex & 5 & Cut-off values for low-risk, intermediate-risk and high-risk groups were not \\
Presence of radiation of pain & 8 & \\
Presence of nausea/sweating & 5 & \\
\hline
\end{tabular}

*Family history of CVD, diabetes mellitus, (treated) hypertension, (treated) hyperlipidaemia, smoking or obesity (body mass index $\geq 30$ ). †CAD, occlusive vascular disease or cerebrovascular disease.

CAD, coronary artery disease; CVD, cardiovascular disease; MI, myocardial infarction.

application (level of evidence is 4). The Gencer rule was developed and externally validated in only one study (C-statistic: $0.75-0.95$, sensitivity $87 \%-98 \%$, specificity $42 \%-71 \%)$. Given the limited evidence, the Gencer rule can only be used with caution (level of evidence for its use is 3 ).
Decision rules designed for acute coronary syndrome

Grijseels et al developed a decision rule for ruling out ACS in general practice in the late 1990s that was later updated by Bruins Slot $e t$ al. These studies show that the discrimination of these decision rules was mediocre (C-statistic of 0.66 and 0.72 ). Unaided clinical judgement provided a better 
Table 3 Diagnostic performance data of the clinical decision rules for coronary artery disease*

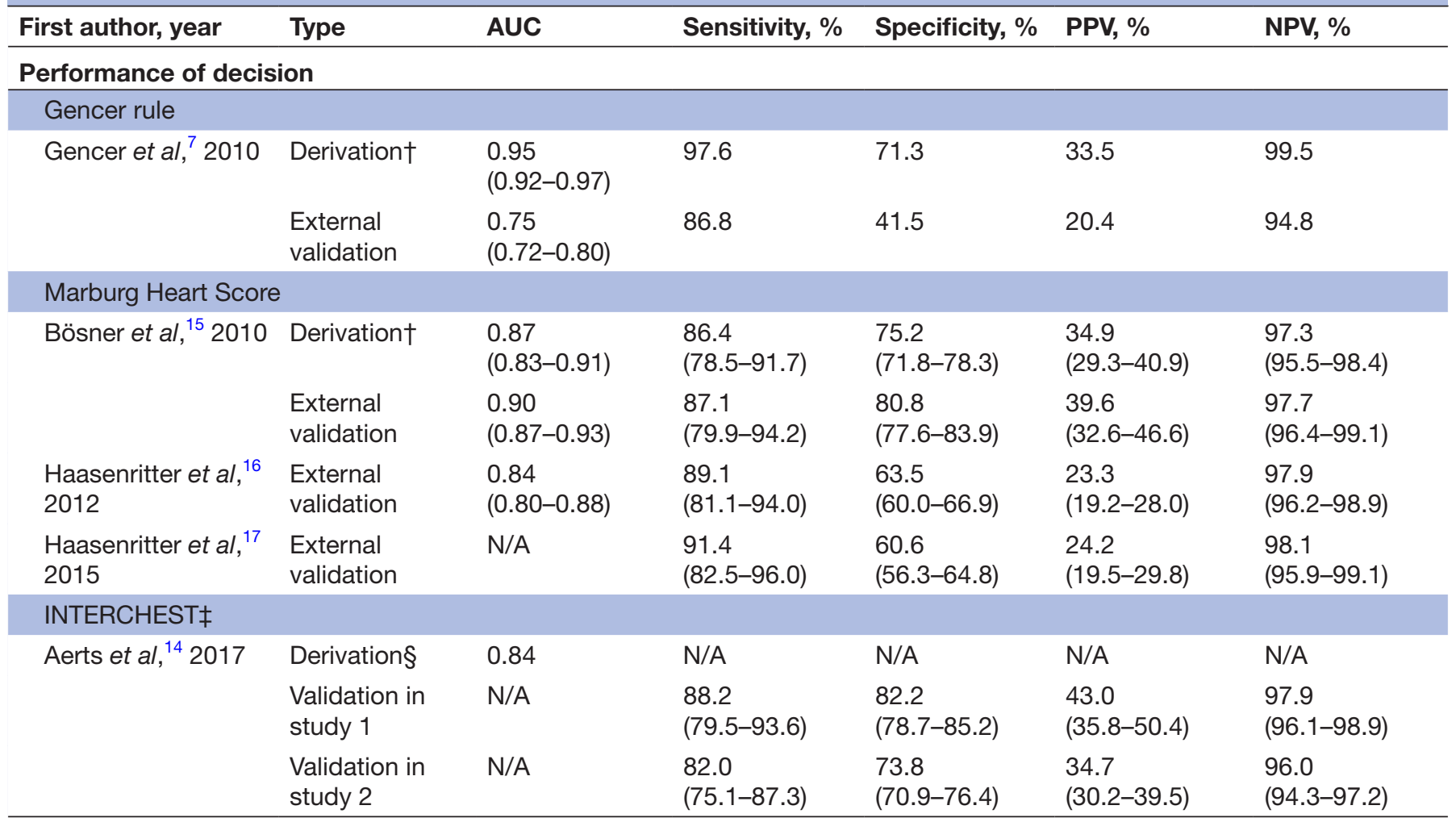

Performance of decision rule versus clinical judgement

Marburg Heart Scoreף

\begin{tabular}{|c|c|c|c|c|c|c|}
\hline \multirow[t]{4}{*}{$\begin{array}{l}\text { Haasenritter et al, }{ }^{17} \\
2015\end{array}$} & $\begin{array}{l}\text { GP's unaided } \\
\text { clinical } \\
\text { judgement }\end{array}$ & N/A & $\begin{array}{l}82.9 \\
(72.4-89.9)\end{array}$ & $\begin{array}{l}61.0 \\
(56.7-65.2)\end{array}$ & $\begin{array}{l}22.7 \\
(18.0-28.2)\end{array}$ & $\begin{array}{l}96.3 \\
(93.6-97.9)\end{array}$ \\
\hline & $\begin{array}{l}\text { Marburg Heart } \\
\text { Score (external } \\
\text { validation) }\end{array}$ & N/A & $\begin{array}{l}91.4 \\
(82.5-96.0)\end{array}$ & $\begin{array}{l}60.6 \\
(56.3-64.8)\end{array}$ & $\begin{array}{l}24.2 \\
(19.5-29.8)\end{array}$ & $\begin{array}{l}98.1 \\
(95.9-99.1)\end{array}$ \\
\hline & $\begin{array}{l}\text { Marburg Heart } \\
\text { Score as triage } \\
\text { test }^{\star \star}\end{array}$ & $\mathrm{N} / \mathrm{A}$ & $\begin{array}{l}81.4 \\
(70.8-88.8)\end{array}$ & $\begin{array}{l}72.6 \\
(68.6-76.3)\end{array}$ & $\begin{array}{l}29.1 \\
(23.2-35.8)\end{array}$ & $\begin{array}{l}96.6 \\
(94.3-98.0)\end{array}$ \\
\hline & $\begin{array}{l}\text { GP's aided } \\
\text { clinical } \\
\text { judgement }\end{array}$ & N/A & $\begin{array}{l}90.9 \\
(72.2-97.5)\end{array}$ & $\begin{array}{l}66.8 \\
(60.5-72.6)\end{array}$ & $\begin{array}{l}20.6 \\
(13.8-29.7)\end{array}$ & $\begin{array}{l}98.7 \\
(95.5-99.6)\end{array}$ \\
\hline
\end{tabular}

*We calculated the sensitivity, specificity, PPV and NPV using two-by-two contingency tables. We used the lowest probability category as 'test negative'.

†Internal validation by means of bootstrapping techniques was performed.

$\ddagger$ Derivation used pooled individual patient data from five studies. The INTERCHEST was applied to two of these five studies to measure its diagnostic performance. We referred to this as 'validation in study 1 and 2'.

§Internal validation by using a threefold cross-validation approach.

IThe GP's unaided clinical judgement was compared with: (1) the Marburg Heart Score; (2) using the Marburg Heart Score as triage test; (3) the GP's clinical judgement aided by the Marburg Heart Score.

${ }^{* *}$ Patients with definite Marburg Heart Score results were counted as negative (score $\leq 2$ points) or positive (score $\geq 4$ points). In patients with an intermediate score (three points), the final test result was determined by the GP's unaided clinical judgement.

AUC, area under the receiver operating characteristic curve; N/A, not applicable; NPV, negative predictive value; PPV, positive predictive value.

overall fit (C-statistic of 0.75 ) with a $51 \%$ agreement in risk estimation. Other diagnostic properties are listed in table 4. Although the study by Bruins Slot is limited by sample size, it appears that the CDR was safer than clinical judgement alone, as four patients that were considered low risk by the GP $(8.2 \%)$ were correctly identified as high risk by the decision aid. The INTERCHEST score was also assessed among 169 patients with acute chest pain; the authors found a reasonable overall performance (C-statistic of 0.79). However, data on its test characteristics were lacking, and as such, we are unable to assess its safety and accuracy. Overall, neither the Grijseels, Bruins Slot or INTERCHEST 


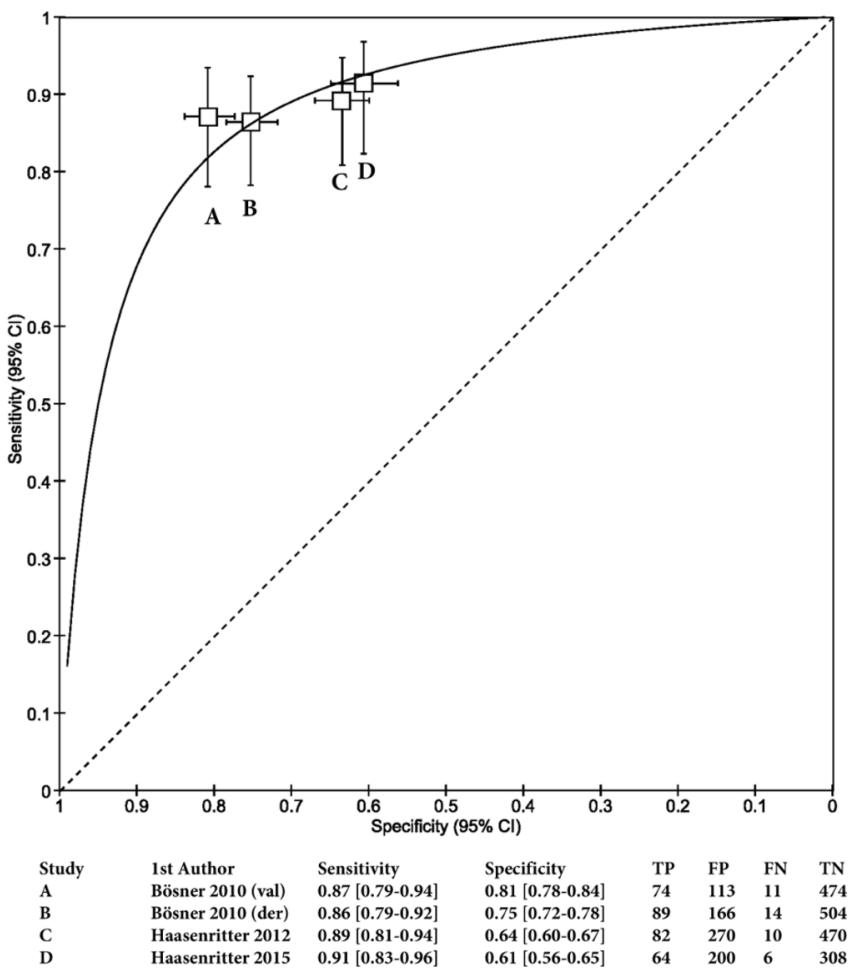

Figure 3 Summary receiver operating characteristic curve of specificity and sensitivity of the Marburg Heart Score across the individual studies.

rules ought to be recommended for rule out of ACS in a general practice setting.

\section{DISCUSSION}

Chest pain presents a diagnostic dilemma in general practice. Advances in therapeutic options, the ageing of our populations and associated increase in patients with chest pain, as well as the fear of medico-legal consequences, have led to a dramatic increase in the number of referrals that threaten to overwhelm the emergency services. ${ }^{18} 19$ CDRs have been coined as an idea to aid in the diagnostic process and to make safe and efficient referral decisions. A prior systematic review on this topic showed that CDRs are not sensitive enough to safely rule out CAD in primary care patients. ${ }^{20}$ We performed an updated systematic review in which we included both derivation, validation and comparative studies with clinical judgement ('gestalt'). Moreover, we made a clear distinction between intermittent-type and acute-onset chest pain, as the diagnostic demands for CDR vary between these two clinical presentations. In summary, we found five primary care-based CDRs that have been developed to differentiate cardiac from non-cardiac chest pain. Three CDRs were developed for ruling out CAD in patients with intermittent chest pain, and two CDRs were developed for patients with symptoms suggestive of ACS. Overall, the Marburg Heart Score holds most promise for ruling out CAD in patients with intermittent chest pain with a consistent, high sensitivity and acceptable specificity and an NPV of $97.3 \%-98.7 \%$ in multiple prospective studies. Moreover, the Marburg Heart Score was more accurate in differentiating CAD from non-CAD than the GP's own clinical judgement, an important argument for implementation into clinical practice. As such, the Marburg Heart score can be used for rule out of CAD in low-risk general practice populations with intermittent-type chest pain (level of evidence of 2). The other CDRs for CAD or ACS lack sufficient validation in external populations or lack sufficient safety or overall accuracy (level of evidence of 3 and 4).

In order for a CDR to be useful in GP settings, it should consist of readily available and/or easy to measure elements. The Marburg Heart Score with its five-item

\begin{tabular}{|c|c|c|c|c|c|c|}
\hline First author, year & Type & AUC & Sensitivity, \% & Specificity, \% & PPV, \% & NPV, \% \\
\hline \multicolumn{7}{|l|}{ Performance of decision } \\
\hline Aerts et al, ${ }^{14} 2017$ & $\begin{array}{l}\text { Sensitivity } \\
\text { analysis }\end{array}$ & 0.79 & N/A & N/A & N/A & N/A \\
\hline Grijseels et al, ${ }^{12} 1996$ & Validation & 0.70 & 91.4 & 36.7 & 56.9 & 82.4 \\
\hline & $\begin{array}{l}\text { GP's aided } \\
\text { clinical } \\
\text { judgement }\end{array}$ & N/A & 97.6 & 21.0 & 53.1 & 90.7 \\
\hline Bruins Slot et al, ${ }^{11} 2011$ & Derivation & $\begin{array}{l}0.66 \\
(0.58-0.73)\end{array}$ & 97.0 & 9.5 & 23.4 & 91.7 \\
\hline
\end{tabular}

AUC, area under the receiver operating characteristic curve; GP, general practitioner; N/A, not available; NPV, negative predictive value; PPV, positive predictive value. 
checklist is both user friendly and seems to do an acceptable job in ruling out CAD in (low-risk) patients with intermittent chest pain. Because of its consistent performance, a point-of-care guide issued by the American Family Physician proposes to integrate the Marburg Heart Score into an algorithm for the evaluation of patients with chest pain in primary care. ${ }^{1}$ It proposes that low-risk patients (score 0 or 1 ) should not receive further cardiac follow-up, whereas high-risk patients $(>3)$ should be referred for cardiac evaluation. In the intermediate/moderate-risk group (score 2 or 3 ), the algorithm proposes the use of the ECG and, when negative, to consult with the cardiologist for further work-up or to order a sequential troponin test. When the troponin test is negative, the risk of a cardiac event is deemed $<1 \%$ within the next 30 days. The guide also states that certain anamnestic elements, including the character of chest pain, should be factored in when making this decision.

While this algorithm may seem appealing, it should be noted that the supportive evidence for the Marburg Heart Score is only applicable for patients with intermittent chest pain in a general practice setting. As such, while risk stratification may be of use to guide referral and diagnostic work-up decisions (ie, exercise testing, etc), there are no data to support the Marburg Heart Score as an ACS rule out tool. This is unfortunate, because it is particularly in the setting of acute-onset chest pain that GPs feel a great need for a CDR. In a recent survey conducted among GPs in the Netherlands, the vast majority of respondents would accept a $<1.0 \%$ risk for missing a diagnosis of ACS in a patient and would accept no more than 25 (in hindsight) unnecessary referrals. ${ }^{21}$ The currently available Grijseels (NPV 82.4\%, PPV $56.9 \%$ ) and Bruins Slot (NPV 91.7\%, PPV 23.4\%) rules fall short of both these targets. The question is whether a CDR based on anamnestic elements will be sufficient to reach a $>99 \%$ NPV. Perhaps, the additional use of pointof-care tests for cardiac markers may increase the safety of a CDR. Studies in general practice found an NPV for troponin and heart-type fatty acid binding protein of $94 \%-96 \%$ for ACS and $99.0 \%-99.7 \%$ for myocardial infarction, respectively. ${ }^{22-26}$ As such, current research efforts focus on whether combining these tests (as pointof-care kits) with a CDR could enhance safety and still provide an effective decision aid. This could be particularly helpful for patients with acute onset of chest pain. Similarly for those with intermittent chest pain, the use of the Marburg Heart Score as a primary care-derived clinical risk assessment tool similar to the Diamond-Forrester chest pain rule ${ }^{27}$ is appealing. However, whether such a strategy is cost-effective compared with usual care should be further evaluated.

\section{Strengths and limitations}

We performed a rigorous systematic search and quality assessment of the included articles involving chest pain rules in primary care. We avoided bias in the selection of studies by two reviewers individually identifying eligible studies, with a third to resolve any disagreements. While not being the first systematic review on this topic, this review is to our knowledge the first that examines the results of the CDRs while taking into account the results of the derivation, validation, and compared the performance of the CDR with the unaided clinical judgement of the GP.

Our study also has a number of limitations. First, we accepted a final diagnosis of coronary artery disease based on a delayed-type reference diagnosis based on consensus of a panel of experts using available symptom-related data and work-up. Such a strategy is valid, as mandating the use of coronary angiography as the reference standard would not be feasible in primary care.$^{28} \mathrm{~A}$ second limitation is the substantial heterogeneity in the prevalence of ACS among studies of CDRs for acute chest pain (range of $22 \%-47.8 \%$ ), which could indicate that GPs may have preselected patients.

Furthermore, we should acknowledge that while we searched for clinical prediction rules for chest pain to rule out CAD or specifically ACS, a minority of patients may present with non-chest pain symptoms (ie, dyspnoea, jaw pain) but do have myocardial ischaemia, these patients (which are more frequently elderly, women and diabetics) may not be properly represented in the included studies. ${ }^{29-31} \mathrm{~A}$ third limitation is that not all included studies reported sufficient data to allow construction of two-by-two contingency tables. Therefore, we cannot accurately assess the performance data of these CDRs. Finally, the CDRs were derived over a span of 22 years. Since the criteria for CAD, the prevalence of risk factors and prevalence of CAD may have changed over the years, some CDRs might be outdated.

\section{Chest pain rules outside primary care}

Our aim was to research the availability of chest pain rules that are applicable and have been validated in low-resource primary care settings. We, therefore, purposefully restricted the scope of this systematic review and excluded CDRs that rely on advanced laboratory, computer or diagnostic testing for their respective scoring systems. We therefore did not include studies on CDRs that are commonly used in EDs, such as the History, ECG, Age, Risk factors and initial Troponin (HEART), ${ }^{32}$ Global Registry of Acute Coronary Events ${ }^{33}$ and Thrombolysis in Myocardial Infarction ${ }^{34}$ scores as well as the more recent Manchester Acute Coronary Syndromes rule. ${ }^{35}$ For a comprehensive overview of chest pain rules recently validated in ED patients, we refer to the systematic review by Liu et al. ${ }^{36}$

\section{Future directions}

Chest pain represents a diagnostic challenge for doctors, particularly in the GP setting, due to an unselected patient population, fewer diagnostic options and time restraints. CDRs may be of assistance, as long as they rely on readily available information and directly applicable. The existing CDRs should be more rigorously tested 
and further optimised, perhaps with the use of machinelearning techniques. Thereafter, we ought to conduct a randomised study in which a CDR-assisted strategy is compared with usual care, in which both safety (clinical outcomes) and efficacy (referral rate) should be assessed. Aside from these research activities, we should also put effort into finding consensus among physicians, patients and other stakeholders in what safety/efficacy balance we are willing to accept when it comes to chest pain. The current trend towards defensive medicine is not sustainable, and as such warrants a discussion on this topic.

\section{CONCLUSION}

Chest pain is a common symptom in primary care, but there is only one validated CDR (Marburg Heart Score) that appears to outperform clinical judgement when applied in patients with intermittent chest pain in a low-risk setting. For ruling out acute coronary syndrome, none of the CDRs was sensitive enough. Future research is warranted for the role of implementing point-of-care cardiac marker tests into CDRs for acute chest pain, as well as the cost-effectiveness of a Marburg Heart Score work-up strategy for intermittent chest pain.

Contributors REH and WAML conceived the study and were responsible for the design and search strategy. REH and SCL were responsible for conducting the search. REH, SCL and JCLH conducted the data analysis and produced the tables and graphs. HCPMvW provided input into the data analysis and interpretation. The initial draft of the manuscript was prepared by REH and SCL then circulated among the coauthors for critical revision. All authors helped to evolve analysis plans, interpret data and critically revise successive drafts of the manuscript.

Funding The authors have not declared a specific grant for this research from any funding agency in the public, commercial or not-for-profit sectors. Funding to cover author processing charges of this publication was provided by the Department of General Practice, Amsterdam UMC, University of Amsterdam, Amsterdam Public Health, Academic Medical Center, Amsterda, The Netherlands.

Competing interests None declared.

Patient consent for publication Not required.

Provenance and peer review Not commissioned; externally peer reviewed.

Data sharing statement There are no additional data available.

Open access This is an open access article distributed in accordance with the Creative Commons Attribution Non Commercial (CC BY-NC 4.0) license, which permits others to distribute, remix, adapt, build upon this work non-commercially, and license their derivative works on different terms, provided the original work is properly cited, appropriate credit is given, any changes made indicated, and the use is non-commercial. See: http://creativecommons.org/licenses/by-nc/4.0/.

\section{REFERENCES}

1. Ebell MH. Evaluation of chest pain in primary care patients. Am Fam Physician 2011;83:603-5.

2. Frese T, Mahlmeister J, Heitzer M, et al. Chest pain in general practice: Frequency, management, and results of encounter. J Family Med Prim Care 2016;5:61-6.

3. Hoorweg BB, Willemsen RT, Cleef LE, et al. Frequency of chest pain in primary care, diagnostic tests performed and final diagnoses. Heart 2017;103:1727-32.

4. McConaghy JR, Oza RS. Outpatient diagnosis of acute chest pain in adults. Am Fam Physician 2013;87:177-82.

5. Nilsson S, Scheike M, Engblom D, et al. Chest pain and ischaemic heart disease in primary care. Br J Gen Pract 2003;53:378-82.
6. Devon HA, Rosenfeld A, Steffen AD, et al. Sensitivity, specificity, and sex differences in symptoms reported on the 13-item acute coronary syndrome checklist. J Am Heart Assoc 2014;3:e000586.

7. Gencer B, Vaucher P, Herzig L, et al. Ruling out coronary heart disease in primary care patients with chest pain: a clinical prediction score. BMC Med 2010;8:9.

8. Moher D, Liberati A, Tetzlaff J, et al. Preferred reporting items for systematic reviews and meta-analyses: the PRISMA statement. BMJ 2009;339:b2535.

9. Whiting PF, Rutjes AW, Westwood ME, et al. QUADAS-2: a revised tool for the quality assessment of diagnostic accuracy studies. Ann Intern Med 2011;155:529-36.

10. McGinn TG, Guyatt GH, Wyer PC, et al. Users' guides to the medical literature: XXII: how to use articles about clinical decision rules. Evidence-based medicine working group. JAMA 2000;284:79-84.

11. Bruins Slot MH, Rutten FH, van der Heijden GJ, et al. Diagnosing acute coronary syndrome in primary care: comparison of the physicians' risk estimation and a clinical decision rule. Fam Pract 2011;28:323-8.

12. Grijseels EW, Deckers JW, Hoes AW, et al. Implementation of a pre-hospital decision rule in general practice. Triage of patients with suspected myocardial infarction. Eur Heart J 1996;17:89-95.

13. Grijseels EW, Deckers JW, Hoes AW, et al. Pre-hospital triage of patients with suspected myocardial infarction. Evaluation of previously developed algorithms and new proposals. Eur Heart $J$ 1995;16:325-32

14. Aerts M, Minalu G, Bösner S, et al. Pooled individual patient data from five countries were used to derive a clinical prediction rule for coronary artery disease in primary care. J Clin Epidemiol 2017;81:120-8.

15. Bösner S, Haasenritter J, Becker A, et al. Ruling out coronary artery disease in primary care: development and validation of a simple prediction rule. CMAJ 2010;182:1295-300.

16. Haasenritter J, Bösner S, Vaucher P, et al. Ruling out coronary heart disease in primary care: external validation of a clinical prediction rule. Br J Gen Pract 2012;62:e415-e421.

17. Haasenritter J, Donner-Banzhoff N, Bösner S. Chest pain for coronary heart disease in general practice: clinical judgement and a clinical decision rule. Br J Gen Pract 2015;65:e748-e753.

18. Schull MJ, Morrison LJ, Vermeulen M, et al. Emergency department overcrowding and ambulance transport delays for patients with chest pain. CMAJ 2003;168:277-83.

19. Smits MF, O.; Weerts M. Spoedritten ambulance vaak eerstelijnszorg. Nederlands Tijdschrift voor Geneeskunde 2014;58:A7863.

20. Ayerbe L, González E, Gallo V, et al. Clinical assessment of patients with chest pain; a systematic review of predictive tools. BMC Cardiovasc Disord 2016;16:18.

21. Harskamp RE, Peet Pvan, Bont J, et al. The conundrum of acute chest pain in general practice: a nationwide survey in The Netherlands, 2018.

22. Bruins Slot $\mathrm{MH}$, Rutten FH, van der Heijden GJ, et al. Diagnostic value of a heart-type fatty acid-binding protein (H-FABP) bedside test in suspected acute coronary syndrome in primary care. Int $\mathrm{J}$ Cardiol 2013;168:1485-9.

23. Bruins Slot MH, van der Heijden GJ, Stelpstra SD, et al. Point-of-care tests in suspected acute myocardial infarction: a systematic review. Int J Cardiol 2013;168:5355-62.

24. Nilsson S, Andersson PO, Borgquist L, et al. Point-of-care troponin T testing in the management of patients with chest pain in the swedish primary care. Int J Family Med 2013;2013:1-7.

25. Planer D, Leibowitz D, Paltiel O, et al. The diagnostic value of troponin $\mathrm{T}$ testing in the community setting. Int $\mathrm{J}$ Cardiol 2006;107:369-75.

26. Tomonaga Y, Gutzwiller F, Lüscher TF, et al. Diagnostic accuracy of point-of-care testing for acute coronary syndromes, heart failure and thromboembolic events in primary care: a cluster-randomised controlled trial. BMC Fam Pract 2011;12:12:12.

27. Diamond GA, Forrester JS. Analysis of probability as an aid in the clinical diagnosis of coronary-artery disease. $N$ Engl J Med 1979;300:1350-8.

28. Knottnerus JA, Muris JW. Assessment of the accuracy of diagnostic tests: the cross-sectional study. J Clin Epidemiol 2003;56:1118-28.

29. Araújo C, Laszczyńska O, Viana M, et al. Sex differences in presenting symptoms of acute coronary syndrome: the EPIHeart cohort study. BMJ Open 2018;8:e018798.

30. Brieger D, Eagle KA, Goodman SG, et al. Acute coronary syndromes without chest pain, an underdiagnosed and undertreated high-risk group: insights from the Global Registry of Acute Coronary Events. Chest 2004;126:461-9.

31. Puymirat E, Aissaoui N, Bonello L, et al. Clinical outcomes according to symptom presentation in patients with acute myocardial 
infarction: Results from the FAST-MI 2010 registry. Clin Cardiol 2017;40:1256-63.

32. Six AJ, Backus BE, Kelder JC. Chest pain in the emergency room: value of the HEART score. Neth Heart J 2008;16:191-6.

33. Granger CB, Goldberg RJ, Dabbous O, et al. Predictors of hospital mortality in the global registry of acute coronary events. Arch Intern Med 2003;163:2345-53.

34. Antman EM, Cohen M, Bernink PJ, et al. The TIMI risk score for unstable angina/non-ST elevation MI: A method for prognostication and therapeutic decision making. JAMA 2000;284:835-42.

35. Body R, Carley S, McDowell G, et al. The Manchester Acute Coronary Syndromes (MACS) decision rule for suspected cardiac chest pain: derivation and external validation. Heart 2014;100:1462-8.

36. Liu N, Ng JCJ, Ting CE, et al. Clinical scores for risk stratification of chest pain patients in the emergency department: an updated systematic review. J Emerg Crit Care Med 2018;2:16. 